\title{
Tracing Oxygen Transport Pathways with In-Situ STEM and Theory
}

Axiel Yaël Birenbaum ${ }^{1 *}$, Valentino R. Cooper ${ }^{1}$ and Albina Borisevich ${ }^{2}$

${ }^{1 .}$ Material Science \& Technology Division, Oak Ridge National Laboratory, Oak Ridge, TN, USA

${ }^{2}$ Center for Nanophase Materials Sciences, Oak Ridge National Laboratory, Oak Ridge, TN, USA

*Currently at Security and Disruptive Technologies, National Research Council, Ottawa, Canada

Energy materials such as oxide-fuel solid cells (SOFC) rely on fast oxygen transport. As oxygen atoms migrate inside the lattice, substantial local distortions are introduced that affect the thermodynamics and kinetics of the overall process. Therefore, detailed understanding of the sequence of events and associated energetics is necessary in order to predict and control transport behaviors, such as anisotropy and defect resistance. Advanced theoretical methods such as Nudged Elastic Band (NEB) [1] can provide a wealth of detailed information on ion trajectories and the energy landscape of the system. However, direct experimental verification is still required to assess impact of e.g. microstructure and defects on the theoretically predicted behavior, such that the material's performance can be improved. In this work, we use NEB calculations in combination with in-situ aberration corrected Scanning Transmission Electron Microscopy (STEM) to reveal details of oxygen transport in $\mathrm{LaCoO}_{3}$ thin films.

Lanthanum cobaltite perovskites exhibit excellent oxygen transport properties [2] and can be grown on a variety of substrates in thin film form to allow for tuning strain conditions [3]. It has also been demonstrated that local oxygen depletion is accompanied by the local expansion of the lattice, which is proportional to the change in the local oxygen content [4], enabling the monitoring of local stoichiometry and lattice configuration with aberration corrected STEM in both static and dynamic [5] settings. Therefore, this system is the perfect test case for the detailed theory-experiment synergy that we seek to demonstrate.

With a combination of NEB approach and STEM measurements we examine the energetics and dynamics of oxygen vacancy transport in $\left(\begin{array}{lll}1 & 1 & 1\end{array}\right)$ oriented $\mathrm{LaCoO}_{3-\delta}$ on $\mathrm{SrTiO}_{3}$.We use the NEB method together with the climbing image technique, solving self-consistently for the energy as implemented in Density Functional Theory. The calculations provide us with step-by-step energetics of the migration process (Fig. 1A) as well as oxygen pathways within the lattice (Fig. 1B) and the associated lattice distortions. We found the migration barriers for an oxygen vacancy to hop from site to site within the tetrahedral layers to be approximately 3.5 $\mathrm{eV}$, and within the center of the octahedral planes $2 \mathrm{eV}$. In contrast, jumping from the apical tetrahedra site to the equatorial octahedral site costs only $1.2 \mathrm{eV}$. We can therefore calculate frequency ratios for oxygen transport events in different directions and compare them to experimental observations.

To acquire STEM data we use a NION® UltraSTEM operated at $200 \mathrm{kV}$. We use the electron beam to induce oxygen motion in $\mathrm{LaCoO}_{3-\delta}$ grown on $\mathrm{SrTiO}_{3}\left(\begin{array}{lll}1 & 1 & 1\end{array}\right)$; changes can be observed on a timescale of $\sim 25$ minutes. We examine the oxygen redistribution by collecting a series of snapshots that are then analyzed to reveal oxygen distributions and lattice distortions as a function of time. Figure 2 illustrates the analysis process; STEM snapshots (Fig. 2A) can be used to compute lattice spacing maps (Fig 2B), which shows the oxygen distribution at a given point in time. Local transport events and associated metastable distortions can be identified by looking at the difference maps between successive snapshots (Fig. 2C), that can be analyzed to reveal correlations between different distortions and to calculate experimental ratios for directional transport. Using pattern recognition approaches to identify specific local configurations revealed by theory (Fig. 1) is also being explored. [6]. 
References:

[1] G. Henkelman and H. Jónsson, J. Chem. Phys. 113, 9901 (2000).

[2] V. S. Bagotsky in "Fuel cells: problems and solutions", ed. ECS, (Wiley, Hoboken NJ)

[2] L. Qiao et al., Nanoletters 154677 (2015).

[4] Y. M. Kim et al, Nature Materials 11 (2012), p. 888.

[5] J. H. Jang et al, ACS Nano 11 (2017), p. 6942.

[6] We thank Prof. Liang Qiao (IFFS, Chengdu, China) for providing the sample. Research was sponsored by the US Department of Energy, Office of Science, Basic Energy Sciences, Materials Sciences and

Engineering Division. This research used resources of the National Energy Research Scientific Computing Center, which was supported by the Office of Science of the US Department of Energy under Contract No. DE-AC02-05CH11231.

A

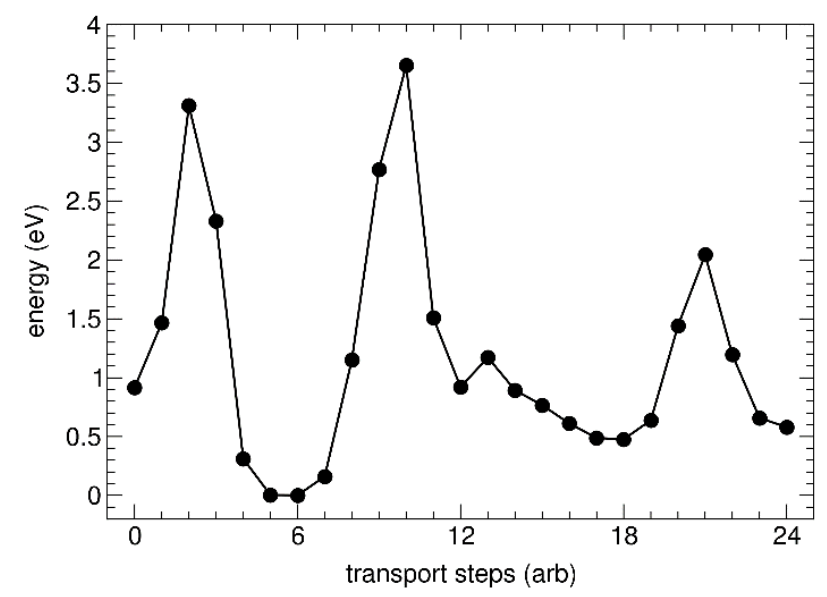

B

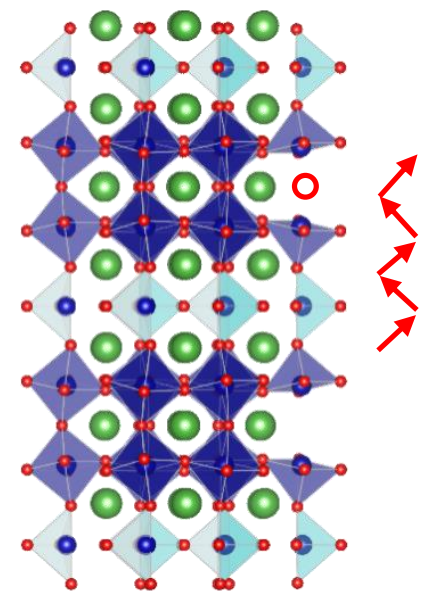

Figure 1. NEB calculation results for oxygen motion through $\mathrm{LaCoO}_{3-\delta}$ : (A) evolution of the total energy of the system, (B) the trajectory of oxygen atom within the lattice.

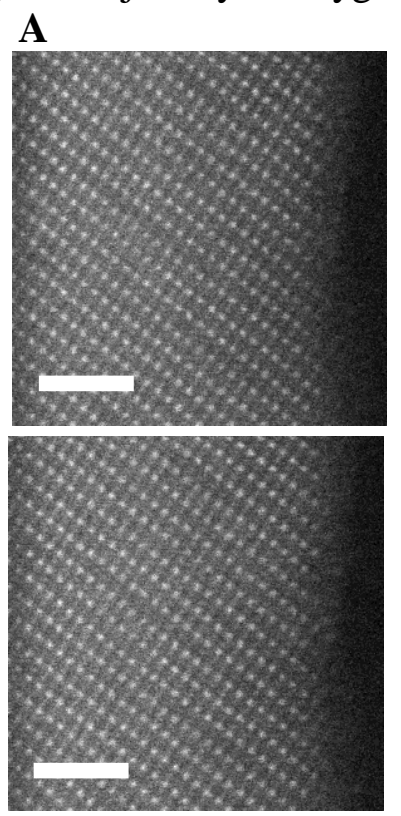

B
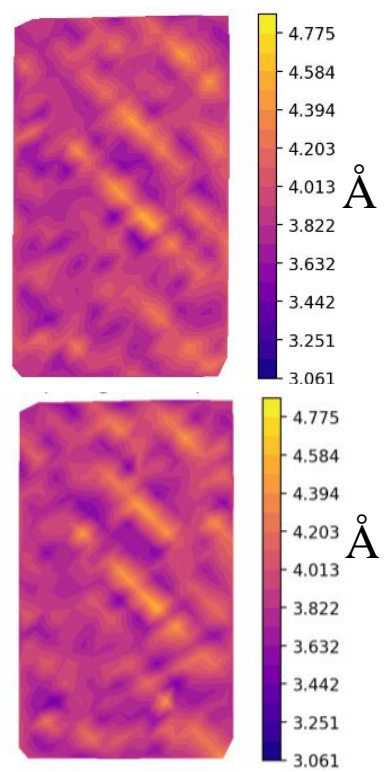

$\mathbf{C}$

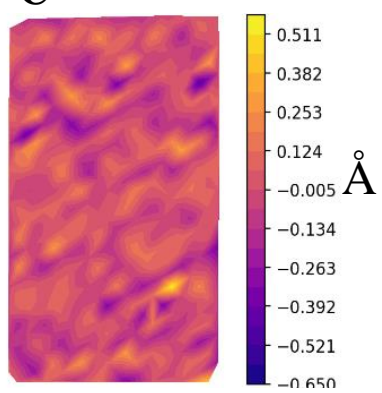

Figure 2. Dynamic data analysis: (A) two subsequent STEM snapshots, (B) the corresponding (100) La-La lattice spacing maps, (C) difference map. Scale bar is $2 \mathrm{~nm}$. 